\title{
Capillary pumped loop for cooling of electronic devices
}

\author{
by T. Wajman, B. Więcek, B. Ostrowski and R. Danych \\ Institute of Electronics, Technical University of Lodz, Poland
}

\begin{abstract}
Capillary pumps are passive, two-phase heat transport systems that use the capillary pressure in a porous material in an evaporator to force circulation of cooling medium flow. The Capillary Pumped Loop (CPL) has been designed, built and investigated. Pump is destined for cooling of electronic component, which work with maximum temperature about $100^{\circ} \mathrm{C}$. Contact surface is $50 \times 50 \mathrm{~mm}$. Investigating the static parameters of the pump is the purpose of this paper. The results indicate that a pump takes off $100-200 \mathrm{~W}$ from the electronic device at $100^{\circ} \mathrm{C}$ near the surface of the power source.
\end{abstract}

\section{Introduction}

Cooling systems play a great role in modern electronics. The increase in power dissipated in electronic elements, together with the progressive miniaturisation, enforces the usage of more and more effective cooling systems [1]. Searching for effective methods of carrying heat away from the semiconductive structure to the ambience is one of the basic issues in electronics. Nowadays, fluid cooling systems, above all those making use of phase transitions of the cooling medium, find new areas of application. Among the efficient solutions of this type, capillary cooling systems (heat pipes [2], loop heat pipes and capillary pumped loops [3, 4]) may be distinguished.

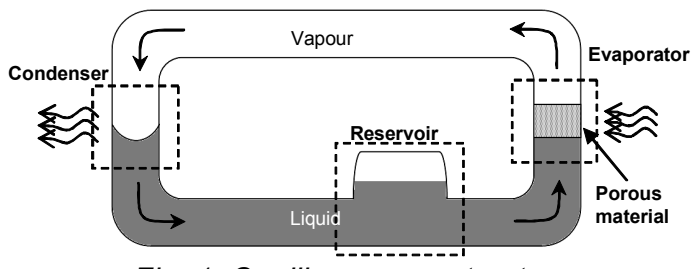

Fig. 1. Capillary pump structure

In the article, a CPL is presented. The diagram of its structure is presented in Fig. 1. There are: an evaporator, a condenser, a reservoir and connecting lines. The evaporator is an element taking the heat directly from the cooling system. It has an inlet of cooled fluid and an outlet of hot vapour. Inside, there is the porous material, which plays a key role in the functioning of the pump. Capillary forces present in porous material cause the underpressure, which sucks in the fluid delivered through the inlet. The fluid is transported onto the surface of the porous material, which is in the direct vicinity of the case. Under the influence of the heat, the cooling medium is evaporating. The vapour is carried away from the surface of the porous material through special grooves to the output. Then, it is transported to the condenser through a vapour line. In the condenser, the heat is dissipated, which causes condensation and cooling down of the medium. The cooled fluid goes back to the evaporator through a separate liquid line. 


\section{Steady state analysis of heat and mass transport in CPL}

In a capillary pump, there is a simultaneous flow of heat and mass. Three phenomena play a basic role: evaporation of fluid from the surface of the porous material, condensation of vapour in the condenser, and a capillary pressure caused by the porous material.

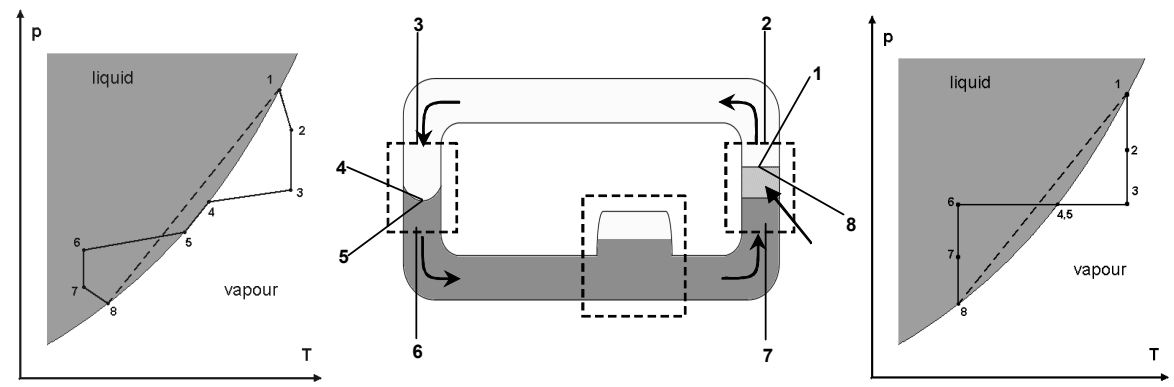

Fig. 2. Full (left), and simplified (right) phase diagram

of the medium in the capillary pump

1, 8 - porous material surface; 2 - vapour outlet; 3 - vapour inlet;

4, 5-condensation surface; 6 - liquid outlet; 7 - liquid inlet.

A phase diagram analysis for the cooling medium is the best way to understand of capillary pumps functioning. In Fig. 2 (left) there is a phase diagram and a chart presenting the capillary pump with corresponding points marked. By analysis of heat and mass transfer in particular elements of the pump, and the interactions between them, the conditions for the pump's operation may be determined.

The total pressure drop of the cooling medium in the pump (from point 1 to 8 ) is the sum of pressure drops in particular elements. After accepting a few assumptions, a simplified model of the cooling medium's flow may be created (fig. 2, right). Firstly, it may be assumed that the temperature of vapour coming out of the evaporator is close to the temperature on the evaporation surface. Then, a negligible temperature change during the medium flow through the liquid and vapour lines may be assumed. Let us as well assume that the pressure drop in the condenser is small compared to the pressure drop in the long vapour and liquid line, and that condensation takes place in a very short section. This all simplifies the phase diagram presented in Fig. 2. Basing on this phase diagram, the pressure drop of the medium in the capillary pump $\left(\Delta \mathrm{p}_{\text {tot }}\right)$ may be determined:

$$
\Delta p_{\text {tot }}=\frac{P}{C_{p}}\left[\frac{1}{\varepsilon A_{v}} \sqrt{\frac{2 \pi R T_{1}}{M}}+\frac{8}{\pi}\left(\frac{v_{v} L_{v}}{R_{v}{ }^{4}}+\frac{v_{l} L_{l}}{R_{l}{ }^{4}}\right)+\frac{v_{l} L_{p}}{A_{v} K}\right]
$$

where: $A_{v}$ - evaporation surface, $M$ - fluid's molar mass, $R$ - the universal gas constant, $\varepsilon$ - evaporation coefficient, $v_{v}, u$ - kinematic viscosity of vapour and liquid, $L_{v}$, $L_{I}-$ the length of vapour and liquid line, $R_{V}, R_{l}-$ the radius of vapour and liquid line, $K$ - the permeability of the porous material, $L_{p}$ - the thickness of the porous material, $P$ - the power carried away by the pump from the cooled system, $T_{1}$ - the evaporation temperature (of the porous material surface), $C_{p}$ - latent heat of evaporation.

The condition for the operation of the CPL is, at least, the balansing of this pressure drop by the capillary pressure of the porous material $\Delta p_{\text {cap }} \geq \Delta p_{\text {tot }}$. The capillary pressure for cylindrical section is expressed as: $\Delta p_{\text {cap }}=2 \sigma \cos \theta / r$, where $\sigma$ - the surface tension, $\theta$ - the contact angle of the fluid with the sides of capillaries, $r$ radius of capillaries. Hence, we have: 


$$
\frac{2 \sigma \cos \theta}{r} \geq \frac{P}{C_{p}}\left[\frac{1}{\varepsilon A_{v}} \sqrt{\frac{2 \pi R T_{1}}{M}}+\frac{8}{\pi}\left(\frac{\eta_{v} L_{v}}{R_{v}{ }^{4} \rho_{v}}+\frac{\eta_{l} L_{l}}{R_{l}{ }^{4} \rho_{l}}\right)+\frac{\eta_{l} L_{p}}{A_{v} K \rho_{l}}\right] .
$$

In the last inequality three unknowns appear: $r, K$ and $\varepsilon$. Parameters of the porous material should be selected in such a way that the inequality (2) has to be satisfied. The value of the evaporation coefficient must be determined experimentally for the specific geometry of the evaporator.

Knowing all parameters of the pump, together with the properties of the porous material and with the evaporation coefficient, one can seek dependencies between the temperature on the surface of the cooled system and on the outlet of the condenser. Assuming the temperature of the condenser one can seek dependencies between the temperature of the surface of the system and the power dissipated in the cooled system.

\section{The prototype of the Capillary Pumped Loop - steady state research}

\subsection{The construction of the pump}

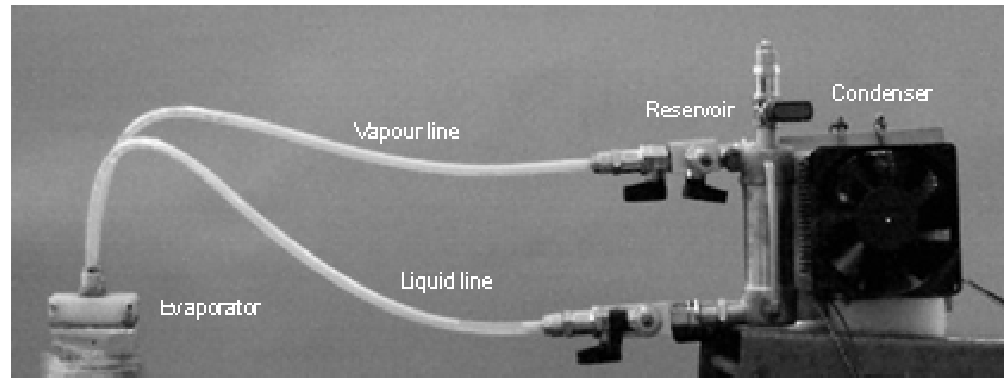

Fig. 3. A prototype of Capillary Pumped Loop

Fig. 3 shows a prototype of CPL constructed in the Institute of Electronics at the Technical University of Lodz. The evaporator's dimensions are: $50 \mathrm{~mm} \times 50 \mathrm{~mm} \times 15 \mathrm{~mm}$. The porous material is a glass-sinter plate of the porosity around 0.727 and permeability: $3.45 \cdot 10^{-10}-1.38 \cdot 10^{-9} \mathrm{~m}^{2}$. The condenser's dimensions are: $100 \mathrm{~mm} \times 100 \mathrm{~mm} \times 20 \mathrm{~mm}$. The reservoir has $90 \mathrm{~mm}$ of height and $30 \mathrm{~mm}$ of diameter. Two semitransparent lines connect the condenser with the evaporator.

\subsection{Results of the pump measurements in steady states}

The temperature of working CPL was measured by infrared camera. It enabled us to find problematic areas, poor contact points, vapour and liquid leakages and other 'hot spots'. Thermal measurements were helpful to monitor of the operation of CPL, if this work steady state. Sample thermograms are shown in Fig. 4. Five measuring points were chosen for contact measurement: the contact surface of the evaporator with the cooling system $\left(T_{s}\right)$, the water inlet $\left(T_{e i}\right)$ and the vapour outlet ( $\left.T_{e o}\right)$ of the evaporator, the vapour inlet $\left(T_{c i}\right)$ and the water outlet $\left(T_{c o}\right)$ of the condenser. Two sets of measurements were executed. In the first set, the natural convection of the condenser radiators was applied. In the second set, the cooling took place through forced convection by means of ventilators. The results of both sets of measurements are presented and discussed below.

In the first set of measurements, the power of the heat source was regulated in the range $75-160 \mathrm{~W}$. Results of measurements are presented in Fig. 5. 

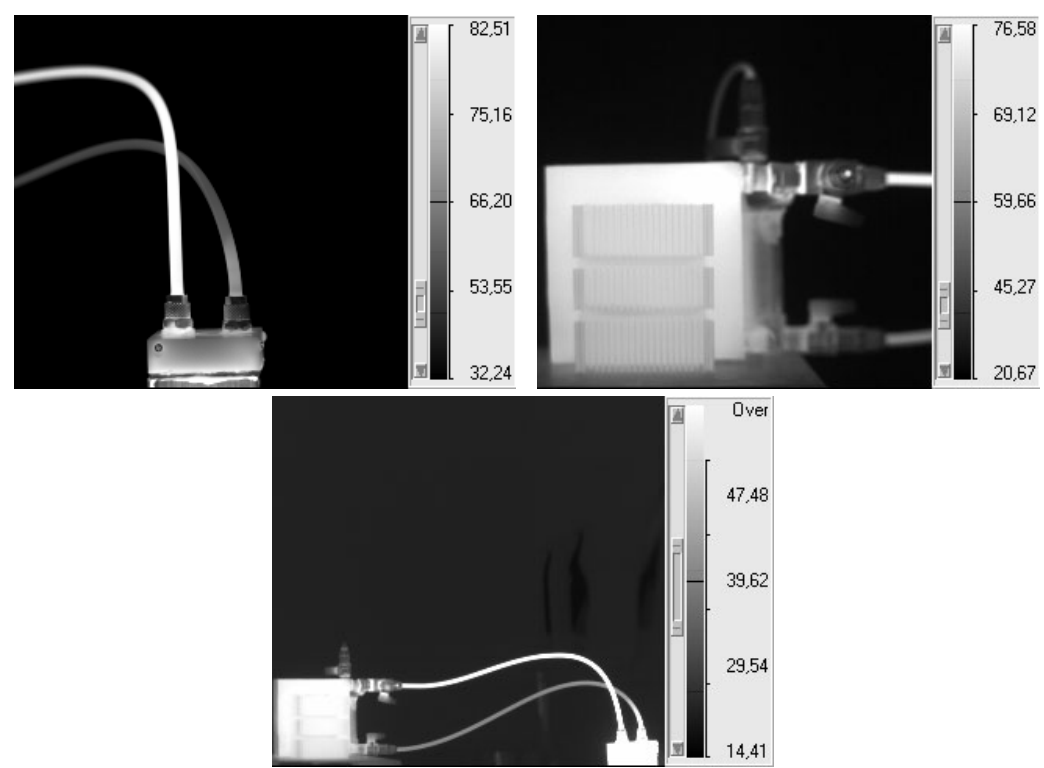

Fig. 4. Sample thermogram of working CPL

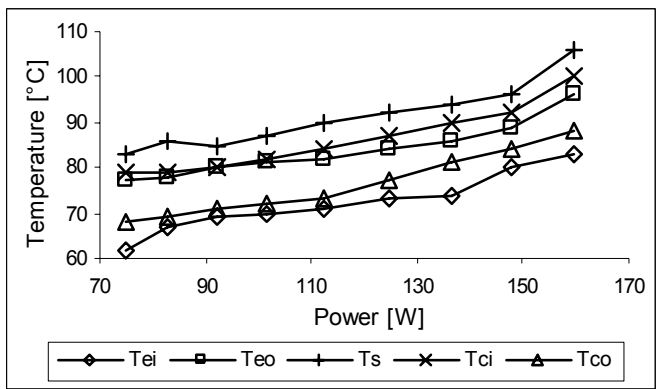

Fig. 5. Test points temperature as the power function for natural convection cooling

According to the assumptions, the pump was to maintain temperature of the surface of the cooled system below $100^{\circ} \mathrm{C}$. The pump complies with the assumptions up to the power slightly exceeding $150 \mathrm{~W}$. Above this limit, temperature of the surface suddenly increases and the capillary pump stops working. It corresponds to reaching the water boiling temperature on the surface of the porous material. It leads to the formation of a considerable quantity of vapour and the disturbance of the circulation of the cooling medium in the pump. During the observation, one could notice a sudden appearance of vapour in the liquid line, a stoppage of the water flow and a sudden increase of temperature of the system surface. The best circulation of the medium occurs for the power from the range 110-150W. Vapour gets out of the evaporator to the vapour line with a high speed and flows into the condenser. After being cooled by about $10^{\circ} \mathrm{C}$ it flows out from the condenser to the evaporator through the reservoir.

In the second set of measurements, forced convection was added to the radiators installed on the condenser. The generated heat was regulated in the range $40-290 \mathrm{~W}$. The results of the temperature measurements are presented in Fig. 6 . 


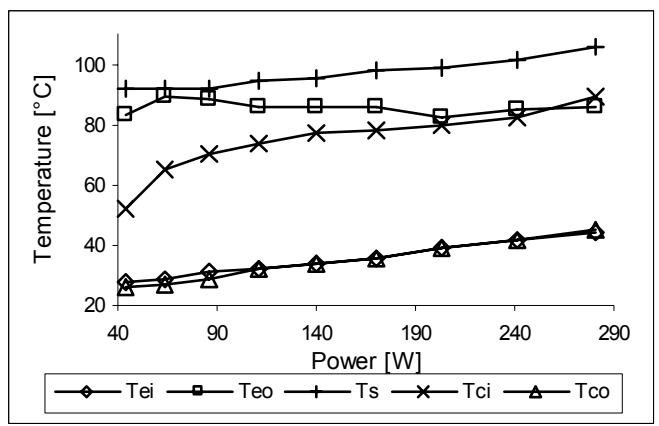

Fig. 6. Test points temperature as the power function for forced convection cooling

With forced convection, the pump maintains temperature of the surface of the cooled system below $100^{\circ} \mathrm{C}$ for the power up to about $230 \mathrm{~W}$. Above this power level, the surface temperature is higher than the assumed. For the power of $230 \mathrm{~W}$, the boiling temperature of water is reached on the surface of the porous material. As previously, it causes formation of a considerable quantity of vapour and the disturbance of the circulation of the cooling medium in the pump. The process was not such impetuous as for the pump with the natural convection cooling. The best circulation of the medium occurs for the power from the range 130-230W. Vapour gets out of the evaporator to the vapour line with a high speed and flows into the condenser. After being cooled by about $40^{\circ} \mathrm{C}$, water flows out of the condenser to the evaporator through the reservoir.

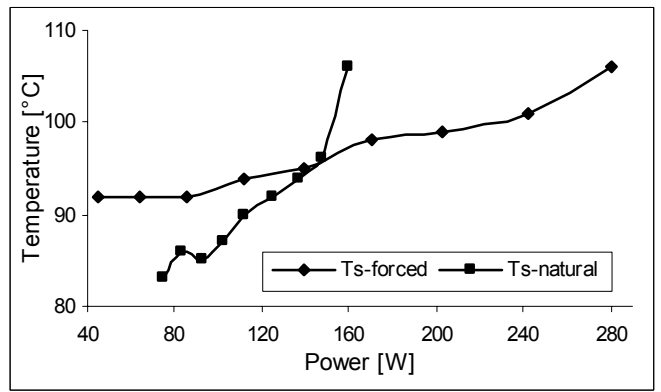

Fig. 7. Results comparison for natural and forced convection cooling

Fig. 7 shows the compared results of the temperature measurement in checkpoints for the system with forced and natural convective cooling (the surface of heat source). It is visible that the system with forced convective cooling operates correctly in the wider range of power generated in the system. It maintains the temperature of the system surface at the established level for considerably greater power than the system with natural convective cooling.

The system with natural convective cooling maintains the required temperature for the smaller range of power. However, as compared with forced cooling for the power to about $150 \mathrm{~W}$, it maintains lower temperature. This difference decreases with the increase of power.

The system with forced convective cooling has smaller dependence between temperature and power dissipated in the device. It is visible that for the system with forced convective cooling, the temperature rise in relation to the increase of power remains almost at the same level of $0 \div 0.2^{\circ} \mathrm{C} / \mathrm{W}$ for the whole range. For natu- 
ral convective cooling, steepness of the characteristics fluctuates considerably in the examined range of power $\left(-0.2 \div 0.8^{\circ} \mathrm{C} / \mathrm{W}\right)$. For the power range $110 \div 150 \mathrm{~W}$, one can notice a small changeability of the characteristics gradient. This is the range in which the stable circulation of the cooling medium in the pump was observed. With the forced convection cooling, stable circulation took place in the wider power range, which is visible by the better stabilization of the system's temperature.

\subsection{Work condition of the pump}

The presented prototype of the pump has the following characteristics:

- the assumed power taken by the pump - 150W,

- the medium - water,

- length of the fluid and water line $-0.5 \mathrm{~m}$,

- the diameter of lines $0.004 \mathrm{~m}$,

- $\quad$ the surface of the porous material $1.26 \cdot 10^{-3} \mathrm{~m}^{2}$ (radius $0.02 \mathrm{~m}$ ),

- the thickness of the porous material $4 \mathrm{~mm}$,

- the temperature of the surface of the porous material $95^{\circ} \mathrm{C}$,

- the temperature of fluid flowing out of the condenser $40^{\circ} \mathrm{C}$.

The porous material used in the capillary pump has porosity 0.727 , permeability range $3.45 \cdot 10^{-10} \div 1.38 \cdot 10^{-9} \mathrm{~m}^{2}$, the diameter of pores $4 \cdot 10^{-5} \div 1 \cdot 10^{-4} \mathrm{~m}$ and the diameter of balls $1 \cdot 10^{-4} \div 2 \cdot 10^{-4} \mathrm{~m}$. After calculating (2), we receive conditions for the value of the evaporation coefficient: $\varepsilon \geq 0.169$ for the minimum permeability and $\varepsilon$ $\geq 0.0812$ for the maximum permeability of the porous material. The evaporator should be characterised by the evaporation coefficient comparable with the one obtained from the calculus.

\section{Conclusions}

During the research described in the article, the simplified static model for the cooling medium in the capillary pump was created. A capillary pumped loop with single porous material was designed and built. The capillary pump asserts carrying away power at the established level of $150 \mathrm{~W}$ from surface of $2.5 \cdot 10^{-3} \mathrm{~m}^{2}$. it gives the heat flux taken from the cooled unit about $60 \mathrm{~kW} / \mathrm{m}^{2}$. In comparison, Astrium's flat CPL [4] can carry away power in the range $0.5 \div 100 \mathrm{~W}$ from surface of $0.9 \cdot 10^{-3} \mathrm{~m}^{2}$, i.e. the heat flux range $0.55 \div 110 \mathrm{~kW} / \mathrm{m}^{2}$.

\section{REFERENCES}

[1] Więcek, B.: Odprowadzanie ciepła w układach elektronicznych ze szczególnym uwzględnieniem konwekcji naturalnej i promieniowania, Zeszyty Naukowe Politechniki Łódzkiej, Nr 820, Łódź (1999)

[2] Legierski, J., Więcek, B.: Heat pipes cooling fins for VLSI integrated circuits, XXII KKTOiUE, Warszawa (1999)

[3] Pin-Chin Chen, Wei-Keng Lin: The application of capillary pumped loop for cooling of electronic components, Applied Thermal Engineering 21, pp. 1739 1754 (2001)

[4] Figus, C., Ounougha, L., Bonzom, P., Supper, W. and Puillet, C.: Capillary fluid loop developments in Astrium, Applied Thermal Engineering 23, pp. 1085 1098, (2003) 